\title{
Langmuir
}

pubs.acs.org/Langmuir

(C) 2010 American Chemical Society

\section{Molecular Friction as a Tool to Identify Functionalized Alkanethiols}

\author{
Joost te Riet, ${ }^{\dagger, \$}$ Tim Smit, ${ }^{\dagger,}$ Jan W. Gerritsen, ${ }^{\dagger}$ Alessandra Cambi, ${ }^{\dagger}$ Johannes A. A. W. Elemans, ${ }^{\dagger}$ \\ Carl G. Figdor, ${ }^{*}$ and Sylvia Speller* ${ }^{*}$ \\ ${ }^{\dagger}$ Department of Scanning Probe Microscopy, Institute for Molecules and Materials, Radboud University \\ Nijmegen, P.O. Box 9010, 6500GL Nijmegen, The Netherlands, and "Department of Tumor Immunology, \\ Nijmegen Centre for Molecular Life Sciences, Radboud University Nijmegen Medical Centre, P.O. Box 9101, \\ $6500 H B$ Nijmegen, The Netherlands. ${ }^{\circledR}$ These authors contributed equally to this Article.
}

Received October 22, 2009. Revised Manuscript Received February 24, 2010

\begin{abstract}
By using the nanografting method, well-defined nanoscale patches of alkanethiols were constructed in a selfassembled monolayer (SAM) matrix on an atomically flat gold $(\mathrm{Au}(111))$ surface. A series of nanografted patches, composed of alkanethiols with different end groups $\left(-\mathrm{CH}_{3},-\mathrm{CF}_{3},-\mathrm{OH},-\mathrm{SH},-\mathrm{COOH}\right.$, and $\left.-\mathrm{NH}_{2}\right)$, were analyzed in detail by a combination of atomic force microscopy (AFM) height and quantitative lateral friction measurements. By constructing a series of nanografted patches of methyl-terminated thiols with various chain lengths, it was shown that the absolute friction of the nanografted patches was always smaller than that of the surrounding SAM matrix, demonstrating that, because of the spatially confined self-assembly during nanografting, SAMs show less defects. In addition, the friction gradually increased for decreasing alkane chain length as expected, although a subtle odd-even effect was observed. The study of thiols with functionalized end groups $\left(-\mathrm{CF}_{3},-\mathrm{OH},-\mathrm{SH},-\mathrm{COOH}\right.$, and $\left.-\mathrm{NH}_{2}\right)$ gave specific insights in orientation, packing, and structure of the molecules in the SAMs. Depending on the thiol end groups, these nanografted patches exhibited large and specific differences in lateral friction force, which offers the unique possibility to use the friction as a molecular recognition tool for thiol-based self-assembled monolayers.
\end{abstract}

\section{Introduction}

In the recent decade, the ability to create structures with dimensions below the micrometer scale has greatly increased the demand for and number of applications of nanosized structures, which requires the development of new methods to manipulate and control the properties of materials down to the molecular level. There are a number of widely available techniques to create well-defined nanoscale structures on surfaces, such as electron beam lithography (EBL), ${ }^{1}$ methods based on chemical vapor deposition (CVD), ${ }^{2}$ and scanning probe microscopy (SPM)assisted lithography, such as dip-pen nanolithography (DPN), ${ }^{3,4}$ nanoshaving, and nanografting. ${ }^{5,6}$ Many of these techniques rely on atomic force microscopy (AFM) for quality control or pattern creation. Since its invention in $1986,{ }^{7}$ AFM has made enormous contributions to the study and development of nanoscale processes, commonly by using it as a topography imaging tool by scanning the sample with a small $(\sim 10 \mathrm{~nm}$ in diameter $)$ tip attached to a cantilever. First introduced by $\mathrm{Xu}$ and $\mathrm{Liu}^{6}$ in 1997, nanografting was presented as a new method for fabricating nanosized structures by using the atomic force microscope not only as an imaging tool, but also as a nanomanipulator to create patterns on a surface using the same cantilever to graft and image. The nanografting technique offers a highly precise control over pattern creation and surface distribution with high lithographic resolution $(\sim 10 \mathrm{~nm}$ and beyond). The versatility of

*To whom correspondence should be addressed. E-mail: s.speller@ science.ru.nl.

(1) Broers, A. N. J. Electrochem. Soc. 1981, 128, 166-170.

(2) Bryant, W. A. J. Mater. Sci. 1977, 12, 1285-1306.

(3) Piner, R. D.; Zhu, J.; Xu, F.; Hong, S. H.; Mirkin, C. A. Science 1999, 283, 661-663.

(4) Salaita, K.; Wang, Y. H.; Mirkin, C. A. Nat. Nanotechnol. 2007, 2, 145-155.

(5) Shi, J. J.; Chen, J. X.; Cremer, P. S. J. Am. Chem. Soc. 2008, 130, 2718-2719.

(6) Xu, S.; Liu, G. Y. Langmuir 1997, 13, 127-129.

(7) Binnig, G.; Quate, C. F.; Gerber, C. Phys. Rev. Lett. 1986, 56, 930-933. a self-assembled monolayer (SAM) of alkanethiols ${ }^{8}$ makes it ideal for pattern transfer., ${ }^{9,10}$ The specific binding to gold $(\mathrm{Au}(111))$ of alkanethiols or other thiolated molecules is enabled via strong and specific sulfur-gold bonds, so that uniform SAMs of high quality are formed. From structural studies by, for example, scanning tunneling microscopy (STM) and reflectance absorption infrared spectroscopy (RAIRS), it is known that alkanethiols form a SAM on a gold surface with their hydrocarbon chains oriented in an alltrans conformation with a lattice constant of $\sim 0.50 \mathrm{~nm}$. ${ }^{6,9,11-16}$ Moreover, they predominantly present a $(\sqrt{ } 3 \times \sqrt{ } 3)$ R3 $30^{\circ}$-based structure (although also a $c(4 \times 2)$ superlattice has been observed) in which the hydrocarbon chains are closely packed and tilted under an angle of $\sim 30^{\circ}$ with respect to the surface normal.

An AFM cantilever is used to nanograft patterns in a SAM matrix by applying a high load force $(>100 \mathrm{nN}),{ }^{17}$ either in contact mode ${ }^{6}$ or in tapping mode. ${ }^{18}$ Under these conditions, the AFM tip locally "shaves" patches of thiol molecules away from the relatively soft SAM matrix (Figure 1). Subsequently, the exposed gold is refilled with other thiol molecules from a supernatant solution. The resulting nanografted patch in the SAM matrix can then be imaged with the same cantilever under a lower

(8) Nuzzo, R. G.; Allara, D. L. J. Am. Chem. Soc. 1983, 105, 4481-4483.

(9) Love, J. C.; Estroff, L. A.; Kriebel, J. K.; Nuzzo, R. G.; Whitesides, G. M. Chem. Rev. 2005, 105, 1103-1169.

(10) Smith, R. K.; Lewis, P. A.; Weiss, P. S. Prog. Surf. Sci. 2004, 75, 1-68.

(11) Fenter, P.; Eberhardt, A.; Eisenberger, P. Science 1994, 266, 1216-1218.

(12) Camillone, N.; Chidsey, C. E. D.; Liu, G. Y.; Scoles, G. J. Chem. Phys. 1993, 98, 3503-3511.

(13) Dubois, L. H.; Nuzzo, R. G. Annu. Rev. Phys. Chem. 1992, 43, 437-463.

(14) Porter, M. D.; Bright, T. B.; Allara, D. L.; Chidsey, C. E. D. J. Am. Chem. Soc. 1987, 109, 3559-3568.

(15) Poirier, G. E.; Pylant, E. D. Science 1996, 272, 1145-1148.

(16) Poirier, G. E.; Tarlov, M. J. Langmuir 1994, 10, 2853-2856.

(17) Castronovo, M.; Bano, F.; Raugei, S.; Scaini, D.; Dell'Angela, M.; Hudej,

R.; Casalis, L.; Scoles, G. J. Am. Chem. Soc. 2007, 129, 2636-2641.

(18) Liang, J.; Scoles, G. Langmuir 2007, 23, 6142-6147.

(19) Liu, M.; Amro, N. A.; Liu, G. Y. Annu. Rev. Phys. Chem. 2008, 59, 367-386. 

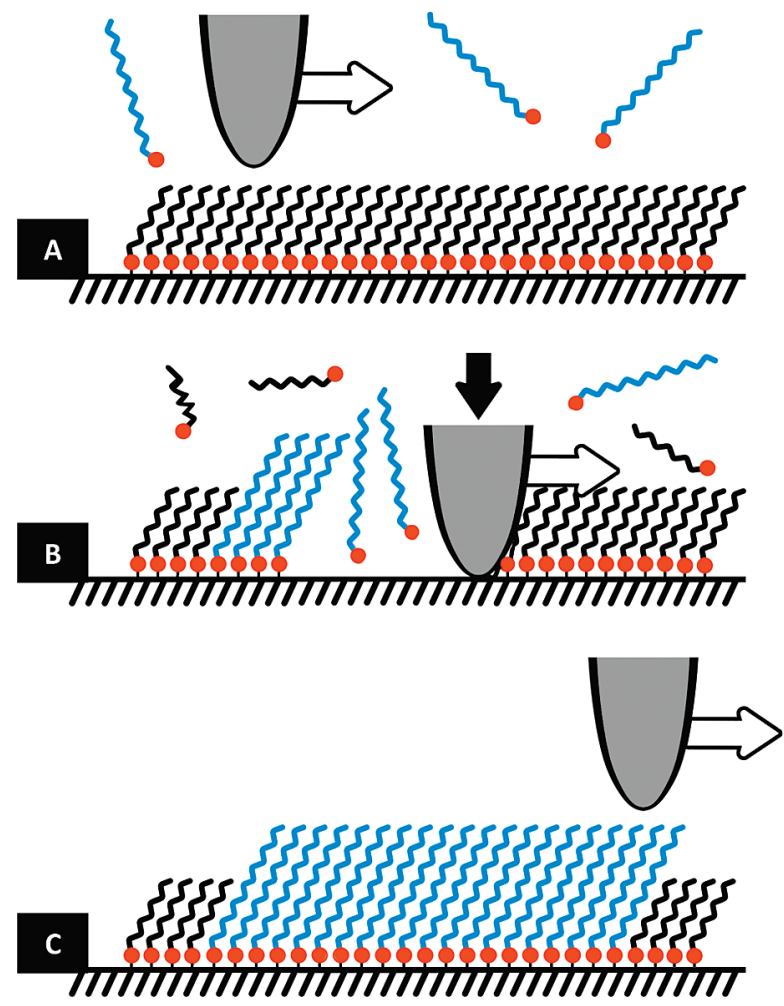

Figure 1. Schematic representation of the nanografting procedure. (A) A Au(111) sample covered with a uniform SAM (in this case $\mathrm{HSC}_{8}$ ) is scanned with AFM under a supernatant solution by scanning with a low contact force $\left(F_{\mathrm{N}} \approx 6 \mathrm{nN}\right)$. (B) By increasing the contact force to $120 \mathrm{nN}$ and increasing the speed, patches of the $\mathrm{SAM}$ can be removed and exchanged by other thiol molecules from solution (in this case $\mathrm{HSC}_{14}$ ). (C) A nanoscale graft has been formed within the matrix SAM which can be imaged again at a low contact force.

load force. This method has been discussed in depth before, ${ }^{6,18,19}$ and with respect to EBL, CVD, or DPN it has many advantages, for example, the ability of working in situ in liquid, without the need for tip modification, and with high accuracy and versatility. Disadvantages of the method are its relatively slow construction speed and small manipulation area, but for studying SAM characteristics at the nanoscale these are of minor importance. Nanografting has provided researchers with new possibilities for the fabrication of nanosized patterns which can be applied to better understand the kinetics of self-assembly, ${ }^{17,20-22}$ but also to specifically organize biomolecules (e.g., proteins and DNA), ${ }^{23-27}$ nanoparticles, ${ }^{28}$ 3D nanostructures, ${ }^{29}$ and bicomponent mixtures. $^{21}$ The availability over internal calibration in situ (the

(20) Xu, S.; Laibinis, P. E.; Liu, G. Y. J. Am. Chem. Soc. 1998, 120, 9356-9361. (21) Yu, J. J.; Tan, Y. H.; Li, X.; Kuo, P. K.; Liu, G. Y. J. Am. Chem. Soc. 2006, $128,11574-11581$

(22) Ivanisevic, A.; McCumber, K. V.; Mirkin, C. A. J. Am. Chem. Soc. 2002, 124, 11997-12001.

(23) Castronovo, M.; Radovic, S.; Grunwald, C.; Casalis, L.; Morgante, M.; Scoles, G. Nano Lett. 2008, 8, 4140-4145.

(24) Chung, S. W.; Presley, A. D.; Elhadj, S.; Hok, S.; Hah, S. S.; Chernov, A. A.; Francis, M. B.; Eaton, B. E.; Feldheim, D. L.; Deyoreo, J. J. Scanning 2008, 30, 159-171.

(25) Mirmomtaz, E.; Castronovo, M.; Grunwald, C.; Bano, F.; Scaini, D.; Ensafi, A. A.; Scoles, G.; Casalis, L. Nano Lett. 2008, 8, 4134-4139.

(26) Staii, C.; Wood, D. W.; Scoles, G. J. Am. Chem. Soc. 2008, 130, 640-646.

(27) Zhou, D. J.; Wang, X. Z.; Birch, L.; Rayment, T.; Abell, C. Langmuir 2003, $19,10557-10562$

(28) Garno, J. C.; Yang, Y. Y.; Amro, N. A.; Cruchon-Dupeyrat, S.; Chen, S. W.; Liu, G. Y. Nano Lett. 2003, 3, 389-395.

(29) Liu, J. F.; Cruchon-Dupeyrat, S.; Garno, J. C.; Frommer, J.; Liu, G. Y. Nano Lett. 2002, 2, 937-940. atomic steps in gold and the matrix SAM) allows quantitative height measurements of the materials involved.

An AFM cantilever tip that scans the topography of a sample in contact mode exerts a load force and gets slightly torsionally deformed while scanning in the lateral direction. This deformation is interpreted as the lateral friction force between tip and sample. This friction is a complex interplay of different physical and chemical factors. The amount of friction the tip experiences depends on the scan speed, the load force $F_{\mathrm{N}}$, the type of probe used, and, most importantly, the composition and structure of the sample. ${ }^{19,30}$ In the case of a surface covered with a SAM, important sample properties are its roughness and packing density, the surface tension, and the number of defects (e.g., crystal edges, grain boundaries, and gauche effects) present in the SAM. ${ }^{9}$ In addition, the nature of the supernatant solution, or the absence of it (i.e., ambient or vacuum), can influence the surface tension between end groups in a SAM and therefore also the friction. ${ }^{17,31}$ Friction mapping can thus provide valuable information about the composition and specific chemical ${ }^{32-37}$ and biological $^{26,38}$ properties of a surface layer at the nanoscale.

In this Article, a combination of nanografting and lateral friction measurements is used to study in a quantitative manner the formation and identity of nanografted SAM patches of alkanethiols with different end groups, such as $-\mathrm{CH}_{3},-\mathrm{CF}_{3}$, $-\mathrm{OH},-\mathrm{SH},-\mathrm{NH}_{2}$, and $-\mathrm{COOH}$. All these measurements are carried out in a liquid environment of 2-butanol, which has the benefit over working in ambient conditions, because the layer on top of the SAM is well-defined and the SAM is in its native state. A thoroughly performed study on methyl-terminated alkanethiols expands the knowledge on the effect of the confined space of nanografting. In particular, the friction changes characteristically. Furthermore, we demonstrate that the ease of using a matrix SAM as internal standard allows to us get direct insight into the orientation and packing of the different (functionalized) alkanethiols within the nanografted patches. In addition, we will show that by using AFM to measure friction it is possible to quantitatively distinguish differences in the chemical nature of the end groups of thiols by measuring their specific frictional properties in a nanografted SAM patch.

\section{Results and Discussion}

Nanografting Alkanethiols in a Self-Assembled Monolayer. Figure 1 shows a cartoon that illustrates the nanografting experiments that have been performed to study functionalized SAMs of alkanethiols. On an ultraflat template-stripped piece of gold (terraces of $200 \times 200 \mathrm{~nm}^{2}$ ), a SAM of $\mathrm{HSC}_{8}$ (see Table 1 for abbreviations used) was grown from a $5 \mathrm{mM}$ solution of the thiol in 2-butanol. After an incubation time of $\geq 18 \mathrm{~h}$, a uniform SAM had been formed. The SAM sample, from now on designated as SAM matrix, was then placed in an AFM liquid-cell containing another thiol in 2-butanol for grafting ( $\mathrm{HSC}_{14}$ in this example). Subsequently, the SAM sample was imaged by AFM in contact mode (Figure 1A), for which a typical force and speed were

(30) Leggett, G. J.; Brewer, N. J.; Chong, K. S. L. Phys. Chem. Chem. Phys. 2005, 7, 1107-1120.

(31) Li, L. Y.; Chen, S. F.; Jiang, S. Y. Langmuir 2003, 19, 666-671

(32) Shuang, L.; Cao, P.; Colorado, R.; Yan, X. P.; Wenzl, I.; Shmakova, O. E.; Graupe, M.; Lee, T. R.; Perry, S. S. Langmuir 2005, 21, 933-936.

(33) Kim, H. I.; Koini, T.; Lee, T. R.; Perry, S. S. Langmuir 1997, 13, 7192-7196. (34) Cruchon-Dupeyrat, S.; Porthun, S.; Liu, G. Y. Appl. Surf. Sci. 2001, 175, 636-642.

(35) Kim, H. I.; Houston, J. E. J. Am. Chem. Soc. 2000, 122, 12045-12046.

(36) Liu, Y. H.; Wu, T.; Evans, D. F. Langmuir 1994, 10, 2241-2245.

(37) Xiao, X. D.; Hu, J.; Charych, D. H.; Salmeron, M. Langmuir 1996, 12, 235 237.

(38) Oncins, G.; Garcia-Manyes, S.; Sanz, F. Langmuir 2005, 21, 7373-7379. 
Table 1. List of Molecules That Were Used for the Formation of SAMs and Nanografted Patches in Our Experiments

\begin{tabular}{|c|c|c|}
\hline chemical name & molecular formula & abbreviated as \\
\hline 1-pentanethiol & $\mathrm{HS}-\left(\mathrm{CH}_{2}\right)_{4}-\mathrm{CH}_{3}$ & $\mathrm{HSC}_{5}$ \\
\hline 1-hexanethiol & $\mathrm{HS}-\left(\mathrm{CH}_{2}\right)_{5}-\mathrm{CH}_{3}$ & $\mathrm{HSC}_{6}$ \\
\hline 1-heptanethiol & $\mathrm{HS}-\left(\mathrm{CH}_{2}\right)_{6}-\mathrm{CH}_{3}$ & $\mathrm{HSC}_{7}$ \\
\hline 1-octanethiol & $\mathrm{HS}-\left(\mathrm{CH}_{2}\right)_{7}-\mathrm{CH}_{3}$ & $\mathrm{HSC}_{8}$ \\
\hline 1-nonanethiol & $\mathrm{HS}-\left(\mathrm{CH}_{2}\right)_{8}-\mathrm{CH}_{3}$ & $\mathrm{HSC}_{9}$ \\
\hline 1-decanethiol & $\mathrm{HS}-\left(\mathrm{CH}_{2}\right)_{9}-\mathrm{CH}_{3}$ & $\mathrm{HSC}_{10}$ \\
\hline 1-undecanethiol & $\mathrm{HS}-\left(\mathrm{CH}_{2}\right)_{10}-\mathrm{CH}_{3}$ & $\mathrm{HSC}_{11}$ \\
\hline 1-dodecanethiol & $\mathrm{HS}-\left(\mathrm{CH}_{2}\right)_{11}-\mathrm{CH}_{3}$ & $\mathrm{HSC}_{12}$ \\
\hline 1-tetradecanethiol & $\mathrm{HS}-\left(\mathrm{CH}_{2}\right)_{13}-\mathrm{CH}_{3}$ & $\mathrm{HSC}_{14}$ \\
\hline 1-pentadecanethiol & $\mathrm{HS}-\left(\mathrm{CH}_{2}\right)_{14}-\mathrm{CH}_{3}$ & $\mathrm{HSC}_{15}$ \\
\hline 1-hexadecanethiol & $\mathrm{HS}-\left(\mathrm{CH}_{2}\right)_{15}-\mathrm{CH}_{3}$ & $\mathrm{HSC}_{16}$ \\
\hline 1-octadecanethiol & $\mathrm{HS}-\left(\mathrm{CH}_{2}\right)_{17}-\mathrm{CH}_{3}$ & $\mathrm{HSC}_{18}$ \\
\hline 6-mercapto-1-hexanol & $\mathrm{HS}-\left(\mathrm{CH}_{2}\right)_{6}-\mathrm{OH}$ & $\mathrm{HSC}_{6} \mathrm{OH}$ \\
\hline 9-mercapto-1-nonanol & $\mathrm{HS}-\left(\mathrm{CH}_{2}\right)_{9}-\mathrm{OH}$ & $\mathrm{HSC}_{9} \mathrm{OH}$ \\
\hline 11-mercapto-1-undecanol & $\mathrm{HS}-\left(\mathrm{CH}_{2}\right)_{11}-\mathrm{OH}$ & $\mathrm{HSC}_{11} \mathrm{OH}$ \\
\hline 1,8-octanedithiol & $\mathrm{HS}-\left(\mathrm{CH}_{2}\right)_{8}-\mathrm{SH}$ & $\mathrm{HSC}_{8} \mathrm{SH}$ \\
\hline $\begin{array}{l}3,3,4,4,5,5,6,6,7,7,8, \\
8,9,9,10,10,10 \text {-heptadecafluor- } \\
\text { 1-decanethiol }\end{array}$ & $\begin{array}{l}\mathrm{HS}-\left(\mathrm{CH}_{2}\right)_{2}- \\
\quad\left(\mathrm{CF}_{2}\right)_{7}-\mathrm{CF}_{3}\end{array}$ & $\mathrm{HSC}_{9}{ }_{9} \mathrm{CF}_{3}$ \\
\hline $\begin{array}{l}\text { 12-mercapto- } \\
\text { 1-dodecanoic acid }\end{array}$ & $\mathrm{HS}-\left(\mathrm{CH}_{2}\right)_{11}-\mathrm{COOH}$ & $\mathrm{HSC}_{11} \mathrm{COOH}$ \\
\hline 11-amino-1-undecanethiol & $\mathrm{HS}-\left(\mathrm{CH}_{2}\right)_{11}-\mathrm{NH}_{2}$ & $\mathrm{HSC}_{11} \mathrm{NH}_{2}$ \\
\hline
\end{tabular}

chosen to avoid compression and deformation of the monolayers (see Experimental Section for details). ${ }^{17}$ Next, an atomically flat part of the surface was selected and a graft was made by shaving the SAM at a high load force, and after that the uncovered gold was immediately filled with thiols from the $\mathrm{HSC}_{14}$ solution (Figure 1B). After the formation of the graft, the sample was imaged at low force again, and features such as height difference between the patch and SAM or lateral force can be determined (Figure 1C).

Quantification of the Height of Nanografted Patches. In Figure 2, AFM images of two $500 \times 500 \mathrm{~nm}^{2}$ nanografted patches are shown, one of a positive (protruding with respect to the background) nanografted patch of $\mathrm{HSC}_{18}$ in a $\mathrm{HSC}_{8} \mathrm{SAM}$ matrix (Figure 2A) and the other one of a negative (lower than background) nanografted patch of $\mathrm{HSC}_{9}$ in a $\mathrm{HSC}_{18}$ matrix (Figure 2B). From the topographies, it is clear that after the grafting procedure other thiols have adsorbed onto the exposed gold, which are most probably those present in the supernatant solution. To confirm this assumption, the height differences between the nanografted patches and the SAM matrix were determined. Relative height differences of $+1.10 \pm 0.18$ and $-1.02 \pm 0.12 \mathrm{~nm}$ were found for the positive and negative grafts, respectively (Figure 2C; see Experimental Section for analysis). These values are in good agreement with the expected differences of +1.08 and $-0.97 \mathrm{~nm}$, which were calculated by the model described in the Experimental Section.

In the topographical images (Figure 2A), atomic gold steps with step sizes of $0.235 \pm 0.06 \mathrm{~nm}$ are clearly visible, values which correspond well to the known $\mathrm{Au}(111)$ step size of $0.235 \mathrm{~nm} .{ }^{39}$ The presence of these steps provides us with a reliable $z$-calibration of the AFM system at the sub-nanometer scale and demonstrates the high quality and accuracy of the measurements.

Quantification of the Friction on Nanografted Patches. When scanning the samples simultaneously with the topography, the friction was probed. In these friction images, the lateral response (torsion) of the AFM cantilever scanning under a $90^{\circ}$ angle with respect to the cantilever long edge axis in contact with

(39) Lang, C. A.; Dovek, M. M.; Nogami, J.; Quate, C. F. Surf. Sci. 1989, 224, L947-L955. the molecular layer is shown. Friction is a unique approach to probe material's and molecular properties at the nanoscale level. In Figure 2D and $\mathrm{E}$, friction images corresponding to the topography images of Figure 2A and B, respectively, are shown, revealing differences in roughness of the SAM matrix with respect to the SAM nanografted patch. In Figure $2 \mathrm{~F}$, cross sections are shown that demonstrate the determination of the net lateral friction. Although coupling between friction and topography cannot be fully excluded, to minimize its influence, the friction of the nanografted patches has been determined in their central region only (see Experimental Section for details). For the nanografted patches shown in Figure $2 \mathrm{~A}$ and $\mathrm{B}$, friction forces of $0.30 \pm 0.09$ and $0.43 \pm 0.06 \mathrm{nN}$ were found for the positive and negative graft, respectively, and $1.05 \pm 0.24$ and $0.60 \pm 0.06 \mathrm{nN}$ for their respective matrices. Remarkably, in both cases, the friction of the grafts was lower than that of the matrix. Furthermore, the friction was lower for progressively longer molecules in the nanografted patches as well as in the matrix, which triggered us to study this property in further detail (see below).

Tilting of Methyl-Terminated Thiols in a Nanografted Patch. By systematically investigating a series of nanografted alkanethiols with a chemically inert methyl end group, the role of the alkane chain length on the topography was elucidated. A full range of $\mathrm{HSC}_{n}$-type molecules was investigated, with their alkane chain length varying from $n=5$ to 18 carbon atoms (Table 1 ), thereby expanding the studies that have been performed earlier by the Liu group. ${ }^{19,40}$ A series of positive $500 \times 500 \mathrm{~nm}^{2}$ nanografted patches was grafted into a $\mathrm{HSC}_{8} \mathrm{SAM}$ matrix, which all formed fully covered patches. By measuring the step size of each of the nanografted patches, relative height differences with respect to the matrix were found ranging from $+1.04 \pm 0.16 \mathrm{~nm}$ for the graft of $\mathrm{HSC}_{18}$ to $-0.25 \pm 0.03 \mathrm{~nm}$ for the graft of $\mathrm{HSC}_{5}$ (Figure $3 \mathrm{~A}$ ). To prove that these relative heights are independent of the surrounding SAM matrix, a control experiment was performed by constructing a series of negative nanografted patches, using the same thiols but now grafted into a $\mathrm{HSC}_{18} \mathrm{SAM}_{\text {matrix. The measured }}$ relative height differences of these grafts with respect to the matrix ranged from $+0.01 \pm 0.09 \mathrm{~nm}$ for $\mathrm{HSC}_{18}$ to $-1.37 \pm 0.09 \mathrm{~nm}$ for $\mathrm{HSC}_{5}$ (Figure 3B). As expected, the relative height difference between the graft and the matrix varies linearly as a function of the length of the molecules for both the positive and negative grafts. The data points were fitted with the expected height differences, as calculated by our model (see Experimental Section) which assumes a tilt angle of $30^{\circ}$ (Figure 3A, B), revealing a good agreement for both the positive and negative grafts $\left(R^{2}=0.990\right.$ and 0.985 , respectively). Alternatively, when the measured data were fitted to obtain the tilt angle, angles of $35.3 \pm 1.3^{\circ}$ and $31.2 \pm$ $1.8^{\circ}$ were found for the positive and negative nanografted patches, respectively. The slightly higher angle calculated for the positive grafts can be explained by collapsing (having a terminal gauche) of the long extending alkanethiols over the edges of the nanografted patch, ${ }^{41}$ in particular those of thiols $\mathrm{HSC}_{14}$ to $\mathrm{HSC}_{18}$ (see Figure $3 \mathrm{~A}$ ). A fit excluding these molecules results in an angle of $30.7 \pm 2.8^{\circ}$, which indicates that our model, assuming a tilt angle of $30^{\circ}$, used to calculate the expected height values is correct. However, most importantly, it demonstrates the high accuracy by which nanografting can be used to determine the orientation of molecules in a SAM.

Friction of Methyl-Terminated Thiols in a Nanografted Patch. In addition to the topography, the friction of the

(40) Xu, S.; Miller, S.; Laibinis, P. E.; Liu, G. Y. Langmuir 1999, 15, 7244-7251.

(41) Price, W. J.; Kuo, P. K.; Lee, T. R.; Colorado, R., Jr.; Ying, Z. C.; Liu, G. Y. Langmuir 2005, 21, 8422-8428. 

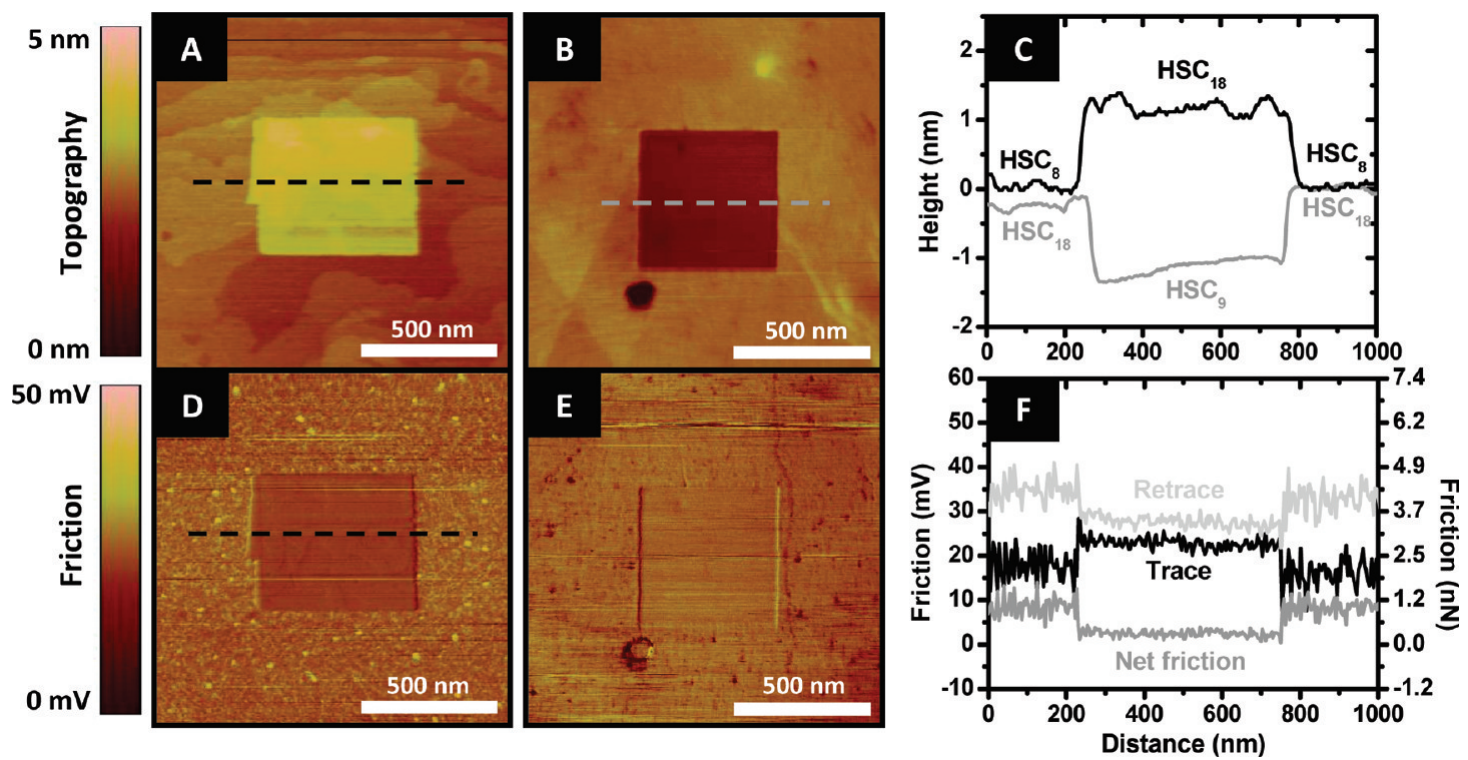

Figure 2. (A) AFM topography image of a freshly nanografted "positive" $\mathrm{HSC}_{18}$ patch grafted in a $\mathrm{HSC}_{8}$ matrix in 2-butanol. The light square represents the nanografted patch, which appears higher in the topography than the surrounding SAM matrix. (B) AFM topography image of a nanografted "negative" $\mathrm{HSC}_{9}$ patch, which appears lower than the surrounding $\mathrm{HSC}_{18}$ matrix. (C) Cross sections of the topography of the nanografted patches, as indicated by the dashed lines in images (A) and (B). (D and E) Friction images (retrace) corresponding to images (A) and (B), respectively. (F) Cross sections of the trace and retrace of the friction channel, as indicated by the dashed line in (D). The raw friction data indicated in black (trace) and light gray (retrace) are subtracted and divided by 2 , yielding a net friction for the $\mathrm{SAM}$ matrix $\left(\mathrm{HSC}_{8}\right)$ of $8.6 \pm 1.9 \mathrm{mV}(1.05 \pm 0.24 \mathrm{nN})$ and for the nanografted patch $\left(\mathrm{HSC}_{18}\right)$ of $2.4 \pm 0.7 \mathrm{mV}(0.30 \pm 0.09 \mathrm{nN})$. All data are \pm SD.
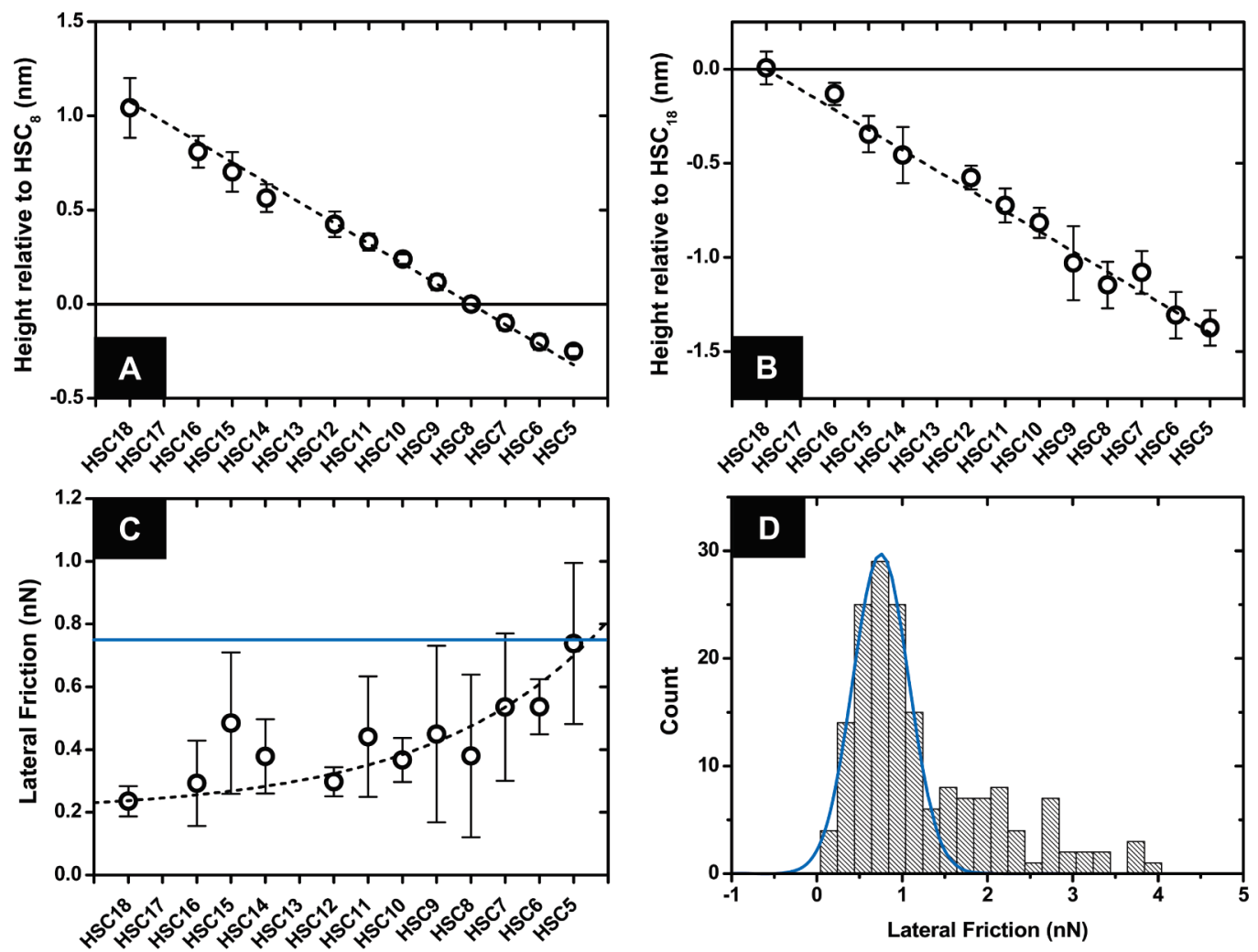

Figure 3. (A) Plot of the heights of "positive" nanografted patches $\left(\mathrm{HSC}_{18}\right.$ to $\left.\mathrm{HSC}_{5}\right)$ grafted into a SAM matrix of $\mathrm{HSC}_{8}$; the dotted line represents the theoretically expected height values. (B) Plot of the heights of the "negative" nanografted patches ( $\mathrm{HSC}_{18}$ to $\mathrm{HSC}_{5}$ ) grafted into a matrix of $\mathrm{HSC}_{18}$. (C) Plot of the friction experienced by the tip while probing the methyl-terminated nanografted patches in a $\mathrm{HSC}_{8}$ matrix. The dotted trendline illustrates the slight increase in friction upon a decrease in thiol chain length. The mean lateral friction of the $\mathrm{HSC}_{8}$ matrix is represented by the solid blue line. (D) Histogram displaying the lateral friction of the SAM matrix (HSC 8 ) for $N=200$ measurements in 2-butanol, measured at a normal force of $6 \mathrm{nN}$ and a scan speed of $\sim 4 \mu \mathrm{m} / \mathrm{s}$. Fitting with a Gaussian (solid blue line) results in a mean lateral friction of $0.75 \pm 0.33 \mathrm{nN}$. Note that the values $>1.5 \mathrm{nN}$ probably represent cases in which measurement conditions were suboptimal, such as sample or tip contaminations. Number of samplings $N \geq 10$, all data $\pm \mathrm{SD}$. 
methyl-terminated nanografted patches and its relation to thiol chain length were investigated in 2-butanol (Figure 3C). Although no large differences in lateral force were measured for the different nanografted $\mathrm{HSC}_{n}$ patches, a clear trend was observed (shown in Figure 3C, dotted trend line): grafts of shorter thiol molecules produce slightly higher friction values than the longer thiols (e.g., $0.24 \pm 0.05 \mathrm{nN}$ for $\mathrm{HSC}_{18}$ and $0.74 \pm 0.26 \mathrm{nN}$ for $\mathrm{HSC}_{5}$ ). This observation can be explained by the higher susceptibility of the grafts of shorter thiols to thermal excitation and pressure exerted by the tip. ${ }^{37,42}$ In AFM friction studies performed under ambient conditions, it was found that the friction for $\mathrm{HSC}_{n}$ molecules rapidly decreases until alkyl chains with a length of $n=12$, after which the friction only slightly decreases. ${ }^{42,43}$ Furthermore, simulations show that gauche effects and kinks decrease in $\mathrm{HSC}_{n}$ SAMs with increasing alkyl chain lengths at least up to a length of $n=16 .^{44,45}$ An important factor is that SAMs of the shorter thiols exhibit a substantial disorder due to less van der Waals interactions, which contribute $4-8 \mathrm{~kJ} \mathrm{~mol}^{-1}$ per methylene group to the overall stabilization of the SAM, ${ }^{9,13,46}$ between their hydrocarbon chains. This facilitates the presence of more SAM and molecular defects (e.g., domain boundaries, missing rows, kinks, and other distortions in the chains) and causes an increased friction probed by the tip that presses on the SAM ${ }^{46}$ However, from our data, it can be concluded that none of the nanografted patches have collapsed and all molecules retain their alignment at a $\sim 30^{\circ}$ tilt with respect to the surface normal (Figure 3A, B). Thus, especially the higher amount of defects in the SAM in the shorter nanografted thiol patches is proposed to cause the higher friction.

In addition to the friction of the nanografted patches, also the friction caused by the $\mathrm{HSC}_{8}$ and $\mathrm{HSC}_{18}$ SAM matrices was analyzed. Figure 3D shows a histogram of the observed lateral friction of the $\mathrm{HSC}_{8}$ matrix, measured over $N=200$ samplings. The normal distribution of the data was fitted with a Gaussian curve, which reveals an average friction of $0.75 \pm 0.33 \mathrm{nN}$. In an analogous manner, an average friction of $0.62 \pm 0.28 \mathrm{nN}$ was found for the $\mathrm{HSC}_{18}$ matrix (data not shown). These observed friction forces, which were determined at $F_{\mathrm{N}} \approx 6 \mathrm{nN}$, correspond well with AFM data previously gathered by Houston et al., ${ }^{47}$ who found a friction of $0.75 \pm 0.15 \mathrm{nN}$ for a $\mathrm{HSC}_{16} \mathrm{SAM}_{\mathrm{St}} 6 \mathrm{nN}$ in 2-butanol. The observed spreading in data (Figure 3D) can be mainly attributed to local variations in the gold substrate and the SAM, but also to variation between AFM cantilevers, especially in the sharpness of the tip.

When the friction values of the nanografted patches, which vary from $0.24 \pm 0.04 \mathrm{nN}\left(\mathrm{HSC}_{18}\right)$ to $0.74 \pm 0.26 \mathrm{nN}\left(\mathrm{HSC}_{5}\right)$, are compared to those of the $\mathrm{HSC}_{8} \mathrm{SAM}$ matrix, they are systematically lower (Figure 3C). This observation indicates that the nanografted patches, in contrast to the matrix SAMs, have a smoother surface, which can be explained by the fact that the selfassembly of the graft thiols during the nanografting is spatially confined. ${ }^{20}$ While grafting, the AFM tip uncovers the gold and allows the thiols present in solution to bind to the surface, a process that occurs $\sim 10$ times faster than the formation of an

(42) Lio, A.; Charych, D. H.; Salmeron, M. J. Phys. Chem. B 1997, 101, 38003805 .

(43) McDermott, M. T.; Green, J. B. D.; Porter, M. D. Langmuir 1997, 13, 25042510.

(44) Alexiadis, O.; Harmandaris, V. A.; Mavrantzas, V. G.; Delle Site, L. J. Phys. Chem. C 2007, 111, 6380-6391.

(45) Alexiadis, O.; Daoulas, K. C.; Mavrantzas, V. G. J. Phys. Chem. B 2008, $112,1198-1211$.

(46) Vericat, C.; Vela, M. E.; Salvarezza, R. C. Phys. Chem. Chem. Phys. 2005, 7, $3258-3568$.

(47) Houston, J. E.; Doelling, C. M.; Vanderlick, T. K.; Hu, Y.; Scoles, G.; Wenzl, I.; Lee, T. R. Langmuir 2005, 21, 3926-3932. ordinary SAM matrix. ${ }^{20} \mathrm{Xu}$ et al. hypothesized that the tip effectively acts as a guide that forces and preorganizes the thiols to be placed in a standing-up configuration. ${ }^{20}$ In contrast, a matrix SAM in an unconstrained environment is formed slowly over a period of hours, allowing the thiols to first adsorb parallel to the surface before they align to the known favorable $30^{\circ}$ orientation. This relatively slow equilibration process not only causes the SAM to have multiple domains in which the thiols adopt an inhomogeneous orientation at the domain boundaries, ${ }^{46}$ but it also results in a larger amount of monolayer defects, ${ }^{20,46}$ which probably contribute to an increase in friction probed by the cantilever. Finally, the friction differences obtained between matrix and nanografted SAM demonstrate in another way the higher ordering of the SAM when it is spatially confined, as proposed by Xu et al. ${ }^{20}$

Odd-Even Effects on the Friction of Methyl-Terminated Thiols in a Nanografted Patch. In Figure 3C, another phenomenon is visible: the friction for grafts of thiol molecules with an odd number of carbon atoms in their chains is slightly higher than that of grafts of thiols with one carbon atom more or less. This is a so-called odd-even effect, ${ }^{9,48}$ which is proposed to be visible in the friction as a result of the difference in orientation of the methyl end groups. Although this odd-even effect is not very pronounced at the used load force of $6 \mathrm{nN}$, we were able to measure it, whereas Mikulski et al. ${ }^{48}$ calculated that a significant odd-even effect would only be noticed at a much higher load force $\left(F_{\mathrm{N}}>50 \mathrm{nN}\right)$. We assume that the high quality of our spatially confined SAMs, as well as the controlled liquid environment, contribute to exhibiting, for the first time, this odd-even effect of methyl-terminated alkanethiols in the friction. Furthermore, it demonstrates the high sensitivity of our method to detect such small differences at the molecular level.

Topography and Friction of SAMs of Thiols with Functional End Groups. Besides the length and packing of the thiol molecules in a nanografted patch, the type of thiol end group (exposed to the tip) will have a profound effect on the tip-sample interaction. ${ }^{49}$ In order to determine this effect, single $500 \times 500 \mathrm{~nm}^{2}$ nanografted patches of thiols of the $\mathrm{HSC}_{n} X$-type, having a variety of functional end groups $\left(X=-\mathrm{CF}_{3},-\mathrm{OH},-\mathrm{SH},-\mathrm{NH}_{2}\right.$, $-\mathrm{COOH})$, were investigated. Each experiment was performed under strictly controlled conditions, with the only variable between the patches being the thiol molecule grafted. In a $\mathrm{HSC}_{8}$ SAM matrix, the following molecules were grafted: $\mathrm{HSC}_{9}{ }_{9} \mathrm{CF}_{3}$, $\mathrm{HSC}_{11} \mathrm{OH}, \mathrm{HSC}_{8} \mathrm{SH}, \mathrm{HSC}_{11} \mathrm{NH}_{2}$, and $\mathrm{HSC}_{11} \mathrm{COOH}$ (Table 1). After imaging, the data from a series of measurements $(N \geq 10)$ were analyzed to obtain the height difference and friction (Figure 4).

SAMs of Fluorocarbon Thiols. For the graft of $\mathrm{HSC}^{\mathrm{F}}{ }_{9} \mathrm{CF}_{3}$ molecules (Figure 4A), a height difference of $+0.37 \pm 0.07 \mathrm{~nm}$ with respect to the matrix was found (Figure 4F), which is in good agreement with the calculated height difference of $+0.39 \mathrm{~nm}$. However, it has to be remarked that $\mathrm{HSC}^{\mathrm{F}}{ }_{9} \mathrm{CF}_{3}$ has not only a $\mathrm{CF}_{3}$ end group but also seven fluorinated carbon atoms extra in its chain (HS- $\left.\left(\mathrm{CH}_{2}\right)_{2}-\left(\mathrm{CF}_{2}\right)_{7}-\mathrm{CF}_{3}\right)$, which influences its SAM formation. A SAM from the fluorocarbon $\mathrm{HSC}^{\mathrm{F}}{ }_{9} \mathrm{CF}_{3}$ is different at some points (crystal structure $c(7 \times 7)$, lattice constant $\sim 0.59$ $\mathrm{nm}$, and chain tilt $\left.16^{\circ}\right)^{50-52}$ from its hydrocarbon counterpart with the same length, $\mathrm{HSC}_{9} \mathrm{CH}_{3}$ (crystal structure $(\sqrt{3} \times$ $\sqrt{ } 3) \mathrm{R} 30^{\circ}$, lattice constant $\sim 0.50, \mathrm{~nm}$ and chain tilt $30^{\circ}$ ).

(48) Mikulski, P. T.; Herman, L. A.; Harrison, J. A. Langmuir 2005, 21, $12197-$ 12206 .

(49) Park, S.; Kim, Y. W.; Lim, J. C.; Ahn, H. S.; Park, S. J. J. Ind Eng. Chem. 2003, 9, 16-24.

(50) Alves, C. A.; Porter, M. D. Langmuir 1993, 9, 3507-3512.

(51) Ostuni, E.; Chapman, R. G.; Holmlin, R. E.; Takayama, S.; Whitesides, G. M. Langmuir 2001, 17, 5605-5620.

(52) Barriet, D.; Lee, T. R. Curr. Opin. Colloid Interface Sci. 2003, 8, 236-242. 


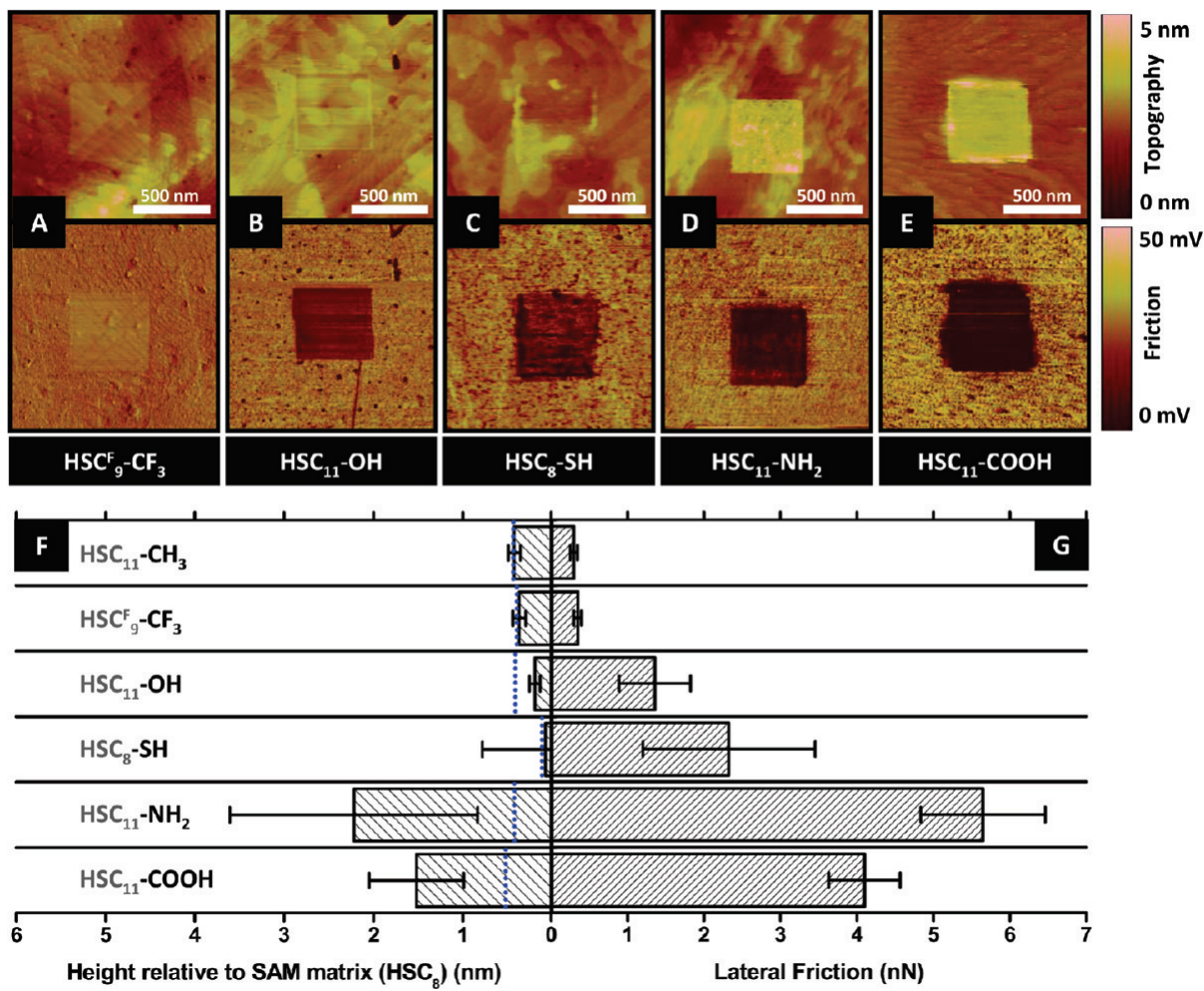

Figure 4. AFM topography (top row) and friction images (retrace, bottom row) of nanografted patches with thiols with various end groups (A) $\mathrm{HSC}_{9}{ }_{9} \mathrm{CF}_{3}$, (B) $\mathrm{HSC}_{11} \mathrm{OH}$, (C) $\mathrm{HSC}_{8} \mathrm{SH}$, (D) $\mathrm{HSC}_{11} \mathrm{NH}_{2}$, and (E) $\mathrm{HSC}_{11} \mathrm{COOH}$. All images were recorded in a 2-butanol supernatant solution, with a normal load force of $\sim 6 \mathrm{nN}$ and at a scan speed of $\sim 4 \mu \mathrm{m} / \mathrm{s}$. Note that for the retrace friction images a darker color implies higher friction. (F) Relative heights, expected height (based on $30^{\circ}$ tilt) indicated by blue dotted line, and (G) corresponding lateral friction values of the different nanografted patches, displayed in bar diagrams. All data are $\pm \mathrm{SD}(N \geq 10)$.

Nevertheless, the lateral friction of $0.35 \pm 0.05 \mathrm{nN}$ (Figure 4G) found for the patch of $\mathrm{HSC}_{9}{ }_{9} \mathrm{CF}_{3}$ is similar to that of $\mathrm{HSC}_{10}(0.37 \pm$ $0.07 \mathrm{nN}$ ). This is a remarkable finding, since in friction studies comparing thiols of the type $\mathrm{HSC}_{n} \mathrm{CF}_{3}$, a molecule with only one fluorinated carbon atom in its end group, and $\mathrm{HSC}_{n} \mathrm{CH}_{3}$ it was found that the friction is higher for the $\mathrm{HSC}_{n} \mathrm{CF}_{3}$-type ${ }^{33,47,53}$ For example, Houston et al. ${ }^{47}$ found a $3-4 \times$ higher friction for $\mathrm{HSC}_{15} \mathrm{CF}_{3}$ than for $\mathrm{HSC}_{15} \mathrm{CH}_{3}$. This observation is, however, explainable: from studies on $\mathrm{HSC}_{n} \mathrm{CF}_{3} \mathrm{SAMs}$, it is known that they display similar lattice constants and structures as their hydrocarbon counterparts of the $\mathrm{HSC}_{n} \mathrm{CH}_{3}$ type. ${ }^{33,52}$ Nonetheless, thiols of the $\mathrm{HSC}_{n} \mathrm{CF}_{3}$-type have larger $\mathrm{CF}_{3}$ end groups than their counterparts $\left(0.57 \mathrm{~nm}\right.$ for $\mathrm{CF}_{3}$ versus $0.42 \mathrm{~nm}$ for $\left.\mathrm{CH}_{3}{ }^{52}\right)$ which will presumably cause discontinuities in the packing of the thiol molecules within the monolayer. We therefore assume that for our measurements the $\mathrm{HSC}^{\mathrm{F}}{ }_{9} \mathrm{CF}_{3}$ molecules within their patch are similarly well-packed as $\mathrm{HSC}_{10}$ molecules, resulting in similar observed friction values, in contrast to the studies on molecules with solely a $\mathrm{CF}_{3}$ end group. However, in comparing these results, one should also consider differences in adhesion between tip and molecules due to difference in surface dipole effects, ${ }^{52}$ which at this stage leaves a direct comparison between our results and those of Houston et al..$^{47}$ unresolved.

SAMs of Hydroxyl-Terminated Thiols. The relative height difference between the nanografted $\mathrm{HSC}_{11} \mathrm{OH}$ patches (Figure $4 \mathrm{~B}$ ) and the $\mathrm{HSC}_{8}$ SAM matrix was determined to be $+0.19 \pm 0.05 \mathrm{~nm}$. This value is significantly lower than the expected value of $+0.42 \mathrm{~nm}$ according to our calculations, which

(53) Kim, H. I.; Graupe, M.; Oloba, O.; Koini, T.; Imaduddin, S.; Lee, T. R Perry, S. S. Langmuir 1999, 15, 3179-3185.
Table 2. Observed Height Differences and Corresponding Tilt Angles for Nanografted Patches of OH-Terminated Alkanethiols

\begin{tabular}{ccc}
\hline type of nanografted patch & $\begin{array}{c}\text { relative } \\
\text { height }(\mathrm{nm})\end{array}$ & $\begin{array}{c}\text { calculated tilt } \\
\text { angle }\left(^{\circ}\right)\end{array}$ \\
\hline $\mathrm{HSC}_{6} \mathrm{OH}$ in $\mathrm{HSC}_{8}$ & $-0.45 \pm 0.12$ & $58.31 \pm 8.13$ \\
$\mathrm{HSC}_{9} \mathrm{OH}$ in $\mathrm{HSC}_{8}$ & $-0.29 \pm 0.08$ & $59.80 \pm 4.01$ \\
$\mathrm{HSC}_{11} \mathrm{OH}$ in $\mathrm{HSC}_{8}$ & $+0.19 \pm 0.15$ & $43.82 \pm 7.98$ \\
& $-1.46 \pm 0.13$ & $53.08 \pm 9.63$ \\
$\mathrm{HSC}_{6} \mathrm{OH}$ in $\mathrm{HSC}_{18}$ & $-1.31 \pm 0.14$ & $56.95 \pm 7.09$ \\
$\mathrm{HSC}_{9} \mathrm{OH}$ in $\mathrm{HSC}_{18}$ & $-0.71 \pm 0.16$ & $29.44 \pm 11.11$ \\
$\mathrm{HSC}_{11} \mathrm{OH}$ in $\mathrm{HSC}_{18}$ &
\end{tabular}

would indicate that the tilt angle of the thiol with the surface normal is higher than the assumed $30^{\circ}$, or collapsing of the thiols on the edges. To further study this behavior and to see if it is dependent on the load force or the length of the thiol, different molecules of the $\mathrm{HSC}_{n} \mathrm{OH}$ type (with $n=6,9,11$ ) were studied. These molecules were grafted into either a $\mathrm{HSC}_{8}$ or a $\mathrm{HSC}_{18} \mathrm{SAM}$ matrix, and the grafts were subsequently imaged in a load force regime of $3-12 \mathrm{nN}$. The observed height differences are summarized in Table 2. By calculating the tilt angle that would correspond to the observed height differences, it turned out that in both SAM matrices the two shorter thiols $\left(\mathrm{HSC}_{6} \mathrm{OH}\right.$ and $\left.\mathrm{HSC}_{9} \mathrm{OH}\right)$ would be tilted more than the $\mathrm{HSC}_{11} \mathrm{OH}$ molecules. This phenomenon had been observed before by Castronovo et al. ${ }^{17}$ who described how hydroxyl-terminated thiols in a nanografted patch could be easily compressed and forced under certain angles with respect to the surface normal. Tilt angles of $43^{\circ}$ and $59^{\circ}$ were found, which is in line with a carbon chain-interlocking model, which roughly describes the

(54) Barrena, E.; Ocal, C.; Salmeron, M. J. Chem. Phys. 2000, 113, 2413-2418. 
quantized tilting of hydrocarbon chains in steps of $\sim 15^{\circ} .{ }^{54,55}$ For our nanografted patches, tilt angles of $53-60^{\circ}$ were calculated for the shorter thiols $\mathrm{HSC}_{6} \mathrm{OH}$ and $\mathrm{HSC}_{9} \mathrm{OH}$, for loads from 3 to $12 \mathrm{nN}$ (see Table 2). Furthermore, we calculated a tilt angle of $\sim 44^{\circ}$ for $\mathrm{HSC}_{11} \mathrm{OH}$ thiols in nanografted patches in a $\mathrm{HSC}_{8}$ SAM matrix and of $\sim 29^{\circ}$ in a $\mathrm{HSC}_{18} \mathrm{SAM}$ matrix, which in the latter case means that the thiols in the grafts are aligned with the matrix molecules and indicates that the surrounding matrix can support the molecules in the grafts. The $\mathrm{HSC}_{11} \mathrm{OH}$ thiols nanografted in the $\mathrm{HSC}_{8}$ matrix molecules stick out above the matrix, so instead of having only a different tilt they can also have collapsed over the edges. ${ }^{41}$

Also the lateral friction of the nanografted patches of the hydroxyl-terminated thiols was measured at different load forces of $3-12 \mathrm{nN}$ and in the two matrix SAMs (Figure 5). At increasing load force, for all nanografted patches, an approximate linear increase in lateral friction was observed, which is, as expected, independent of the SAM matrix (data not shown). Typically, at a load of $6 \mathrm{nN}$, the friction values measured for the grafts are about 3 times higher than those measured for grafts of thiols with methyl end groups. In analogy with the trend observed for nanografted patches of methyl-terminated thiols, the friction increases slightly with decreasing thiol length. In line with earlier observations, we propose that the higher compressibility and decrease in packing of the SAM, induced by, for example, end group size and electrostatic interactions between the end groups, is an important cause for the $3 \times$ higher friction.

SAMs of Dithiols. The nanografted patch of dithiol $\mathrm{HSC}_{8} \mathrm{SH}$ (Figure 4C) exhibits a relative height difference of $+0.13 \pm 0.65$ $\mathrm{nm}$ with respect to the matrix, which at first glance corresponds well with the theoretical height difference of $+0.15 \mathrm{~nm}$. However, the large variation in the measurement indicates a decreased smoothness when compared to that of the earlier described nanografted patches. Explanations for this relative roughness might be the connection of two or more $\mathrm{HSC}_{8} \mathrm{SH}$ molecules through intermolecular $\mathrm{S}-\mathrm{S}$ bonds, ${ }^{56,57}$ and the binding of both thiol groups of the same molecule to the gold surface. ${ }^{57}$ As a consequence, the friction value of the $\mathrm{HSC}_{8} \mathrm{SH}$ graft $(2.32 \pm 1.13 \mathrm{nN}$, Figure $4 \mathrm{G})$ is $5 \times$ higher than that observed for $\mathrm{HSC}_{9}$. In addition, throughout the graft, the friction is not fully uniform, which also corroborates the previously mentioned disorders in the patch.

SAMs of Amine- and Acid-Terminated Thiols. Finally, the characteristics of nanografted patches containing amine- and acid-terminated alkanethiols were determined (Figure 4D, E). Height differences of $+2.22 \pm 1.39$ and $+1.52 \pm 0.53 \mathrm{~nm}$ were measured between the $\mathrm{HSC}_{8}$ matrix and the $\mathrm{HSC}_{11} \mathrm{NH}_{2}$ and $\mathrm{HSC}_{11} \mathrm{COOH}$ grafts, respectively. These height differences are much larger than expected because calculations predicted values of only +0.42 and $+0.52 \mathrm{~nm}$ for $\mathrm{HSC}_{11} \mathrm{NH}_{2}$ and $\mathrm{HSC}_{11} \mathrm{COOH}$, respectively. A plausible explanation for this observation can be the occurrence of bilayer formation via intermolecular hydrogen bonding between the amine or carboxylic acid groups, as has also been observed by Wang et al. ${ }^{58}$ These findings will be described and discussed in more detail elsewhere. ${ }^{59}$ The measured lateral forces of $5.65 \pm 0.81 \mathrm{nN}$ for the graft of $\mathrm{HSC}_{11} \mathrm{NH}_{2}$ and $4.10 \pm$ $0.47 \mathrm{nN}$ for the graft of $\mathrm{HSC}_{11} \mathrm{COOH}$ both show a huge increase (19-fold and 14-fold, respectively) in friction compared to that of the graft of methyl-terminated thiols with a similar length $\left(\mathrm{HSC}_{12}\right)$. It is assumed that this increase in friction cannot solely

(55) Barrena, E. Ocal, C. Salmeron, M. J. Chem. Phys. 2001, 114, 4210-4214.

(56) Liang, J.; Rosa, L. G.; Scoles, G. J. Phys. Chem. C 2007, 111, 17275-17284

(57) Yu, J. J.; Ngunjiri, J. N.; Kelley, A. T.; Garno, J. C. Langmuir 2008, 24, $11661-11668$.

(58) Wang, H.; Chen, S. F.; Li, L. Y.; Jiang, S. Y. Langmuir 2005, 21, 2633-2636.

(59) te Riet, J. Unpublished results.

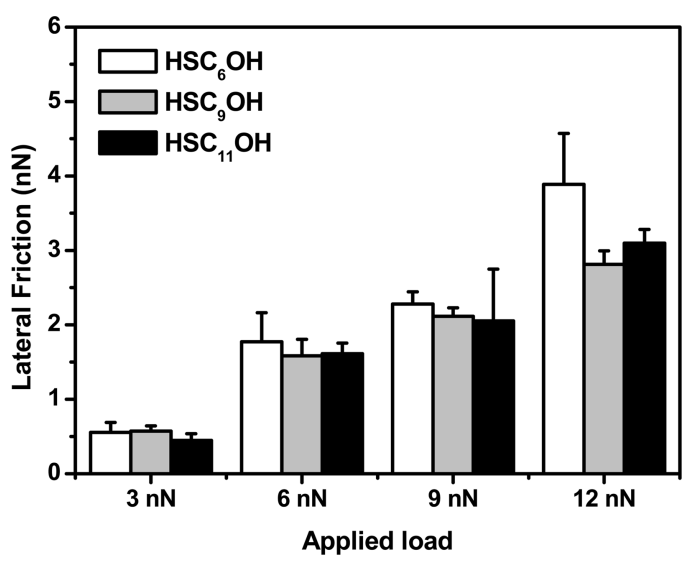

Figure 5. Lateral friction experienced by the AFM tip of nanografted patches of hydroxyl-terminated alkanethiols $\left(\mathrm{HSC}_{n} \mathrm{OH}\right)$ with different chain lengths $(n=6,9,12)$, measured at varying applied load forces of 3-12 $\mathrm{nN}$ in 2-butanol and with a scan speed of $\sim 4 \mu \mathrm{m} / \mathrm{s}$. Data have been collected on nanografted patches in both a $\mathrm{HSC}_{8}$ and $\mathrm{HSC}_{18}$ SAM matrix. All data are $\pm \mathrm{SD}(N \geq 20)$.

be attributed to the difference in end group but is mainly caused by the formation of the bilayer structure. We propose that the second layer, connected to the underlying layer via hydrogen bonds, is less resistant to pressure exerted by the tip and has more defects.

Concluding Remarks on Nanografted Functional Alkanethiols. In conclusion, the friction values of the nanografted patches composed of thiols with functional end groups are higher than those of grafts of methyl-terminated thiols. Considering the similarity of the thiol alkyl chain backbones of the different thiols, the differences in friction might be attributed to the size of the end group as well as to interactions between the end groups, for example, hydrogen bonds, and their impact on intermolecular packing of the thiols. Obviously, these parameters are not independent, and at this stage we cannot discriminate properly between the different factors that contribute to the overall friction. In particular, the case of $\mathrm{HSC}^{\mathrm{F}}{ }_{9} \mathrm{CF}_{3}$ illustrates the effect of chain packing on the friction. If this packing is suboptimal, like in the case of fluoro/hydrocarbon chains, the friction increases. Furthermore, for thiols with functionalized end groups, there is probably also an effect induced by interactions between end groups and AFM tip (e.g., hydrophobic/hydrophilic) or by specific interactions between the end group and the solvent. The latter factor is of course very important, because it can, for example, be expected that a relatively polar solvent such as 2butanol will interact more favorably with polar thiol end groups than an apolar solvent, and this difference might have a profound effect on the friction properties of a monolayer. Therefore, although we expect that the friction tool can also be successfully applied to differentiate between nanografted patches of different thiols in other solvents, each of these systems should be investigated separately in order to establish their specific friction characteristics.

Recognition of the Functional End Groups in Multigrafts. The ability to recognize grafts of functionalized alkanethiols by their topographical and frictional "signatures" opens the possibility to use AFM as a true surface identification tool. To investigate this, a set of grafts, each of which contains thiols with a different end group, was created within the same $\mathrm{HSC}_{8} \mathrm{SAM}$ matrix. Figure 6A shows an AFM image of such a multigraft with nanografted patches of $\mathrm{HSC}_{11} \mathrm{NH}_{2}, \mathrm{HSC}_{11} \mathrm{OH}$, and $\mathrm{HSC}_{18}$ thiols. It was constructed by rinsing the sample in situ after 


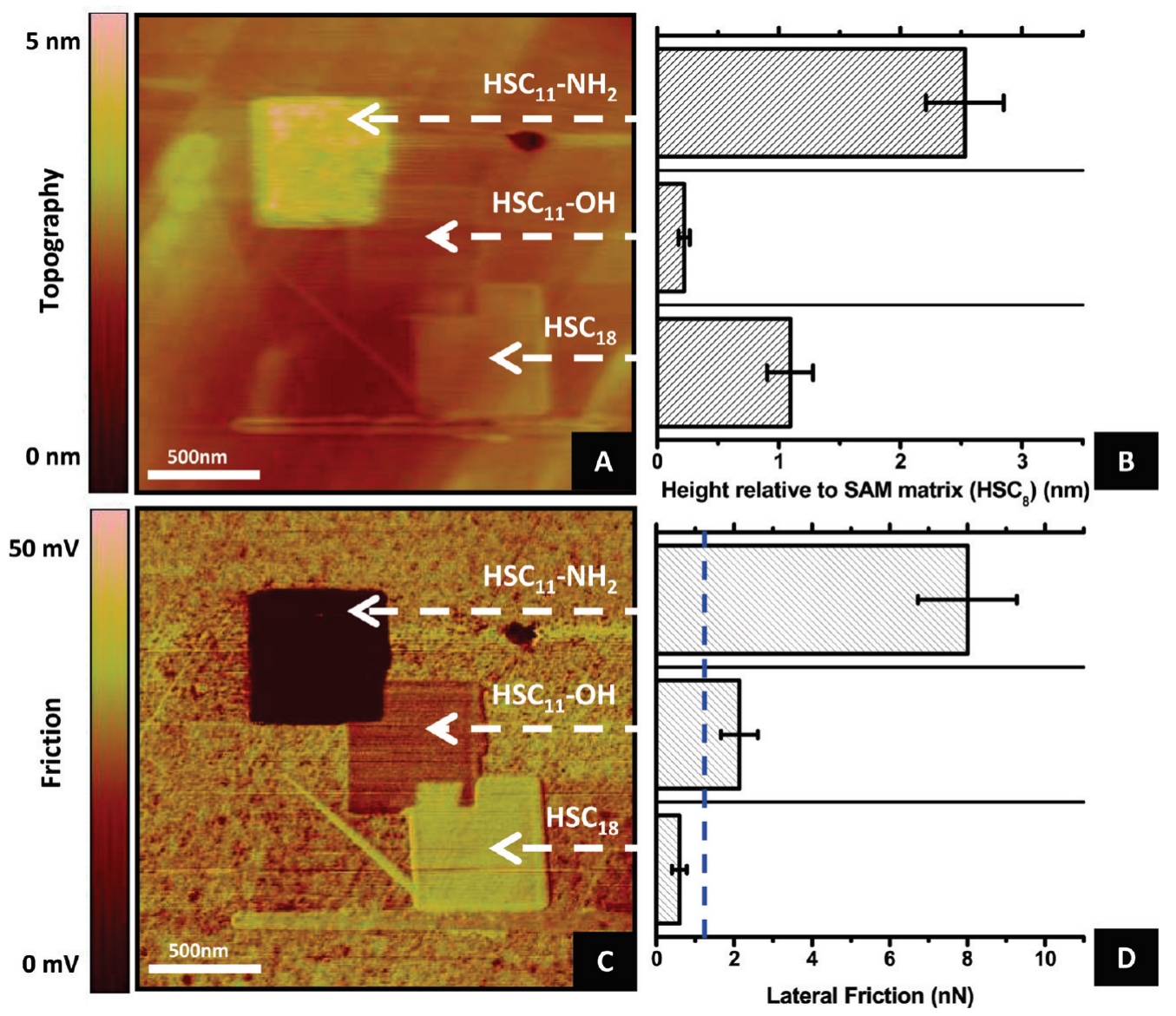

Figure 6. (A) AFM topography image of a multigraft of $\mathrm{HSC}_{18}, \mathrm{HSC}_{11} \mathrm{OH}$, and $\mathrm{HSC}_{11} \mathrm{NH}_{2}$ (in creation order) recorded at load force of

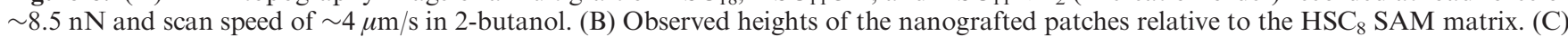
Corresponding friction (retrace) image showing the different friction signatures of the grafts of $-\mathrm{CH}_{3^{-}},-\mathrm{OH}-$, and $-\mathrm{NH}_{2}$-terminated thiols $(N=10)$. (D) Average lateral friction values of the nanografted patches. The friction of the SAM matrix is indicated by the blue dashed line $(1.21 \pm 0.14 \mathrm{nN})$. All data are $\pm \mathrm{SD}$.

creating each nanografted patch. Subsequently, the nanografting procedure was repeated at a different spot at the sample with a supernatant solution containing a different thiol. Height differences with respect to the matrix of $+1.09 \pm 0.19,+0.22 \pm 0.05$, and $+2.53 \pm 0.32 \mathrm{~nm}$ were measured for the nanografted patches of $\mathrm{HSC}_{18}, \mathrm{HSC}_{11} \mathrm{OH}$, and $\mathrm{HSC}_{11} \mathrm{NH}_{2}$, respectively. These values are similar to those determined earlier for the single nanografted patches. However, more interestingly, the corresponding friction image (Figure 6C) clearly reveals a different friction "signature" for each of the nanografted patches. Values of $0.6 \pm 0.2,2.1 \pm 0.5$, and $8.0 \pm 1.3 \mathrm{nN}$ were obtained for the grafts of $\mathrm{HSC}_{18}$, $\mathrm{HSC}_{11} \mathrm{OH}$, and $\mathrm{HSC}_{11} \mathrm{NH}_{2}$, respectively (Figure 6D). These results nicely demonstrate that the frictional "signatures" of the nanografted patches (ratio 1:3:14) constitute a useful source of identification.

By comparing the multigraft with the single nanograft experiments, it can be safely concluded that in both cases the observed lateral friction differences are caused by inherent differences in graft properties, which are directly related to their molecular structure and not by experimental variables between individual measurements. However, more interestingly, it is clear that with the described method nanografted patches of thiols with different end groups can be specifically recognized by probing their friction.

\section{Conclusions}

From a series of well-defined nanografted patches of methylterminated alkanethiols with various lengths, height differences of single carbon atoms were observed, implying a sub-nanometer precision in height. The measurements further revealed that in both positive and negative nanografted patches the thiols are oriented in an all-trans configuration under an angle of $30^{\circ}$ with respect to the surface normal, which extends on results of earlier studies. Quantitative lateral force measurements showed that the friction of the nanografted patches was always lower than that of the surrounding matrix SAM. This effect can be attributed to the spatially confined self-assembly process during nanografting described by $\mathrm{Xu}$ et $\mathrm{al}^{20}{ }^{2}$ and is clear evidence of the fact that the topographical roughness is directly correlated to this process. Furthermore, the friction of the nanografted patches decreased with increasing thiol length, which is mainly attributed to differences in packing of hydrocarbon chains. The ability to probe a subtle odd-even effect on the friction highlights the sensitivity of the method for detecting differences in molecular packing within a monolayer on the sub-nanometer scale.

The same techniques were applied to analyze SAMs of thiols with a variety of functional end groups. While the topographies of the nanografted patches composed of thiols with $-\mathrm{CF}_{3}$ and $-\mathrm{SH}$ end groups were as expected, the grafts of thiols with $-\mathrm{OH}$ end groups appeared to be compressed, with the thiol molecules tilted under angles of $\sim 43^{\circ}$ and $\sim 59^{\circ}$, depending on their chain length. This SAM compression is explained by hydroxyl end group induced surface tension and weaker van der Waals interactions, resulting in an increase in disorder in the packing of the molecules in the nanografted patches. Strong indications were found for the 
formation, via hydrogen bonding interactions, of bilayers in the nanografted patches constructed of thiols with $-\mathrm{NH}_{2}$ and $-\mathrm{COOH}$ end groups. For each of the nanografted patches, highly characteristic friction values were found, which increase for thiols with end group $\mathrm{CH}_{3} \leq \mathrm{CF}_{3}<\mathrm{OH} \leq \mathrm{SH}<\mathrm{COOH} \leq \mathrm{NH}_{2}$. This has opened the possibility of using the lateral friction as a unique recognition tool to identify the building blocks of a nanografted patch, which was confirmed by the fact that multiple nanografted patches of thiols with different end groups in the same matrix SAM could be accurately assigned by their specific friction characteristics.

\section{Experimental Section}

Chemicals Used and SAM Preparation. All chemicals used (Table 1) were purchased from Sigma-Aldrich (St. Louis, MO) and used as received unless stated otherwise. Thiol solutions for SAM formation and grafting were prepared in $>99.5 \%$ 2-butanol. The SAMs were prepared on an ultraflat patch of template-stripped gold, which was prepared according to the procedure described by Hegner et al. ${ }^{60}$ In short, it can be summarized in three steps: (i) First, a $0.25 \mathrm{~cm}^{2}$ piece of mica coated with a $300 \mathrm{~nm}$ thick layer of gold (Georg Albert PVDBeschichtungen, Heidelberg, Germany) was glued to a clean glass slide, with its gold side pointing downward, using a two-component epoxy glue (type 377, Epoxy Technology Inc., Waterloo, Belgium), leaving the mica side exposed to air. (ii) Subsequently, the sample was heated at $150{ }^{\circ} \mathrm{C}$ for $2 \mathrm{~h}$, which activates the hardening process of the glue. (iii) Finally, the sample was submerged in a solution of tetrahydrofuran (THF) for $5 \mathrm{~min}$, after which it can be stripped at the gold-mica interface, removing the mica. After stripping, the sample was immediately submerged in a freshly prepared solution of $5 \mathrm{mM}$ of alkanethiol in 2-butanol. After incubation for $\geq 18 \mathrm{~h}$ in the thiol solution, ${ }^{9}$ the gold sample was washed with ethanol $(99.8 \%)$ and dried with a gentle flow of nitrogen.

Atomic Force Microscopy. Imaging and nanografting were performed using two Veeco MultiMode atomic force microscopes with a Nanoscope IV and V controller (Veeco Industries, Santa Barbara, CA), both equipped with an E-type piezo scanner ( $X Y$ range $\sim 12 \mu \mathrm{m}$ ) and a liquid-cell (MTFML, Veeco) holding the cantilever. The AFM system was calibrated by using a $1 \times 1 \mu \mathrm{m}^{2}$ (100 $\mathrm{nm}$ deep) calibration grid. After calibration, the sample was placed in the liquid-cell and subsequently submerged in a $50 \mu \mathrm{L}$ droplet of a $5 \mathrm{mM}$ 2-butanolic solution of an alkanethiol. The whole sample and droplet were then enclosed by a fluorosilicate O-ring (FSFCO-10, Veeco). A laser beam was focused on the end point of a V-shaped $\mathrm{Si}_{3} \mathrm{~N}_{4} \mathrm{NP}$-S cantilever (Veeco; cantilever A on the NP-S chip, nominal spring constant $0.58 \mathrm{~N} / \mathrm{m}$ ), which had been cleaned by rinsing with chloroform and by irradiation with UV light (20 min.). Moreover, directly prior to every experiment, the cantilever was rinsed with ethanol $(99.8 \%)$. The laser beam was deflected onto a four-quadrant photosensitive detector; the monitored vertical deflection was interpreted as the topography, while the horizontal deflection, caused by the torsional deformation of the cantilever when its tip scans over the surface, was interpreted as the lateral friction force between tip and sample.

Imaging was performed in contact mode, under a low load force of $F_{\mathrm{N}} \approx 6 \mathrm{nN}(\sim 1 \mathrm{~V}$ deflection set point $)$ at $1 \mathrm{~Hz}(\sim 4 \mu \mathrm{m} / \mathrm{s}$ for a $2 \times 2 \mu \mathrm{m}^{2}$ image). The pressure that the tip then exerts is $\sim 0.5 \mathrm{GPa}$, as calculated by the Hertzian model ${ }^{54}$ assuming a tip radius of $15 \mathrm{~nm}$ and a contact area of $12 \mathrm{~nm}^{2}$. Nanoshaving of the SAM was performed at a high load force of $F_{\mathrm{N}} \approx 120 \mathrm{nN}(\sim 20 \mathrm{~V})$ at $15 \mathrm{~Hz}(\sim 60 \mu \mathrm{m} / \mathrm{s})$, and the exerted pressure was $\sim 10 \mathrm{GPa}$.

Data and images were analyzed by using NanoScope 6.13 and 7.20 (for NS IV and V) and Origin 8 software. The friction was determined from both trace and retrace of the lateral force images;

(60) Hegner, M.; Wagner, P.; Semenza, G. Surf. Sci. 1993, 291, 39-46. these data were analyzed off-line by subtracting both channels (raw data), dividing by 2 for averaging, and subsequently converted into friction data $(\mathrm{nN})$ using the method outlined below. For statistics, the height data were analyzed by taking consecutive cross sections (sampling over 20-50 lines) via step size determination on the edges of the nanografted patch on a single gold terrace within the software. Per image, $N \geq 5$ of these samplings were performed. Subsequently, at least three images per nanografted patch were analyzed per experiment (up and down scans), and also at least three independent experiments in total (all with different cantilevers, to exclude the possibility that differences in friction are caused by differences in tip shape). The friction was determined only in the $450 \times 450 \mathrm{~nm}^{2}$ center region of the $500 \times$ $500 \mathrm{~nm}^{2}$ nanografted patches to eliminate edge effects. Furthermore, the friction was collected for the same amount and set of images as the topography analysis with a sampling of three on every image. In addition to the friction measurements of the nanografted patches, in all experiments, also the friction of the SAM matrix was determined in order to ascertain the quality and reliability of the measurements and, when necessary, to exclude measurements from further analysis. For all analyzed data, errors given are \pm SD.

Quantifying the AFM Data. V-shaped NP-S cantilevers (Veeco) were calibrated combining the Sader method ${ }^{61}$ and the thermal oscillation method, ${ }^{62}$ with some minor practical adaptations. In short, the vertical, torsional, and lateral spring constants of the V-shaped cantilever were determined by the following method. The vertical spring constant was determined by using the included thermal tune software module of the NS V system (Veeco) by fitting the primary resonance peak with the simple harmonic oscillator model. ${ }^{63}$ The system can process the data for resonances up to $100 \mathrm{kHz}$. ${ }^{64}$ After a deflection sensitivity (from now designated as InvOLS; inverse optical lever sensitivity) calibration in air of the cantilever mounted in the setup $(N=$ 5), we used the Hutter and Bechhoefer method, ${ }^{62}$ with the later described corrections for a V-shaped cantilever, ${ }^{64,65}$ to determine the vertical spring constant. The torsional and physically related lateral spring constant for a V-shaped cantilever was calculated by using the parallel beam approach for a composite ceramic-gold cantilever. $^{66-69}$ The following formulas were used to calculate the lateral spring constant,

$$
k_{\text {lat }}=\frac{4}{3 \cos ^{2} \theta+6(1+v) \sin ^{2} \theta}\left(\frac{L}{H}\right)^{2} k_{z}
$$

and the torsional spring constant,

$$
k_{\text {tors }}=k_{\text {lat }} H^{2}
$$

where $\theta$ is the inner angle between the cantilever beam and the substrate, $v$ is the Poisson ratio, $L$ is the length of the cantilever, $H$ is the height of the tip, and $k_{z}$ is the vertical spring constant. For the used NP-S cantilever (cantilever A of the chip), the (nominal) dimensions given by the manufacturer are $\theta=62^{\circ}, v=0.24$ (for $\left.\mathrm{Si}_{3} \mathrm{~N}_{4}\right),{ }^{70} L=115 \mu \mathrm{m}, w=25 \mu \mathrm{m}$ (width), $H=3 \mu \mathrm{m}, \Delta L=4 \mu \mathrm{m}$ (tip set-back), $t=0.6 \mu \mathrm{m}$ (thickness), and $R=10-20 \mathrm{~nm}$ (tip radius). We determined an overall vertical spring constant

(61) Sader, J. E.; Larson, I.; Mulvaney, P.; White, L. R. Rev. Sci. Instrum. 1995 $66,3789-3798$

(62) Hutter, J. L.; Bechhoefer, J. Rev. Sci. Instrum. 1993, 64, 1868-1873.

(63) Sader, J. E.; Chon, J. W. M.; Mulvaney, P. Rev. Sci. Instrum. 1999, 70, 3967-3969.

(64) Ohler, B. Rev. Sci. Instrum. 2007, 78, 0637011-5.

(65) Stark, R. W.; Drobek, T.; Heckl, W. M. Ultramicroscopy 2001, 86, 207-215. (66) Noy, A.; Frisbie, C. D.; Rozsnyai, L. F.; Wrighton, M. S.; Lieber, C. M. J. Am. Chem. Soc. 1995, 117, 7943-7951.

(67) Sader, J. E. Rev. Sci. Instrum. 1995, 66, 4583-4587.

(68) Sader, J. E.; Sader, R. C. Appl. Phys. Lett. 2003, 83, 3195-3197.

(69) Hazel, J. L.; Tsukruk, V. V. Thin Solid Films 1999, 339, 249-257.

(70) Alexander, W.; Shackelford, J. F. CRC Materials Science and Engineering Handbook; CRC Press/Taylor and Francis: Boca Raton, FL, 2001; Vol. 3, p 537. 
of $k_{z}=252 \pm 19 \mathrm{pN} / \mathrm{nm}( \pm \mathrm{SD} ; N=6$; all chips from one wafer $)$ by the method described above (note the $57 \%$ difference with the manufacturer's data). By using the formulas above, we calculated a lateral spring constant of $k_{\text {lat }}=159 \pm 20 \mathrm{~N} / \mathrm{m}$ and a torsional spring constant of $k_{\text {tors }}=(2.06 \pm 0.15) \times 10^{-9} \mathrm{Nm} / \mathrm{rad}$. We determined a vertical InvOLS for the setup in 2-butanol (note: different from that in $\left.\mathrm{air}^{71}\right)$ of $23.76 \pm 0.43 \mathrm{~nm} / \mathrm{V}( \pm \mathrm{SD})$ and a horizontal InvOLS of $0.77 \pm 0.10 \mathrm{~nm} / \mathrm{V}( \pm \mathrm{SD})$, which leads to an overall conversion rate for the photodetector data (in volts) to the contact force (in newtons) of $5.98 \pm 0.46 \mathrm{nN} / \mathrm{V}$ and for the friction force of $0.123 \pm 0.017 \mathrm{nN} / \mathrm{mV}$.

Molecular Model. The apparent height of the molecules in the SAM was calculated using data from literature; ${ }^{72}$ bond lengths are $0.1523 \mathrm{~nm}(\mathrm{C}-\mathrm{C}), 0.2293 \mathrm{~nm}(\mathrm{~S}-\mathrm{Au}), 0.1815 \mathrm{~nm}$ $(\mathrm{C}-\mathrm{S}), 0.1338 \mathrm{~nm}(\mathrm{C}-\mathrm{O}), 0.1208 \mathrm{~nm}(\mathrm{C}=\mathrm{O})$, and $0.1438 \mathrm{~nm}$

(71) Tocha, E.; Song, J.; Schönherr, H.; Vancso, G. J. Langmuir 2007, 23, 70787082 .

(72) Lide, D. R. Handbook of Chemistry and Physics, 88th ed.; CRC Press/Taylor and Francis: Boca Raton, FL, 2007-2008.
$(\mathrm{C}-\mathrm{N})$ and the angle between the $\mathrm{Au}-\mathrm{S}-\mathrm{C}$ and $\mathrm{C}-\mathrm{C}-\mathrm{C}$ bonds in the alkyl backbone is in both cases $109.5^{\circ}$. With these data, the expected lengths of the alkanethiols were calculated using ChemBio3D Ultra 11.0. Subsequently, by taking into account a $30^{\circ}$ tilt (if not stated otherwise) for the molecules, ${ }^{9,14}$ the apparent height or thickness of the layer is calculated.

Acknowledgment. J.t.R. is supported by NanoNed, the Dutch nanotechnology programme of the Ministry of Economic Affairs. A.C. is supported by a VENI grant (916.66.028) of The Netherlands Organization for Scientific Research (NWO). J.A.A.W.E. thanks the Council for the Chemical Sciences of The Netherlands Organization for Scientific Research (CW-NWO) for a VIDI grant. C.G.F. is supported by a BIO-LIGHT-TOUCH grant (FP6-2004-NEST-C-1-028781) and Immunanomap grant (MRTN-CT-2006-035946) of the European Union. Arend M. van Buul is acknowledged for kindly providing us with 11-amino1-undecanethiol. 